\title{
Correction: CUL4B promotes gastric cancer invasion and metastasis-involvement of upregulation of HER2
}

M. Qi, M. Jiao, X. Li, J. Hu, L. Wang, Y. Zou, M. Zhao, R. Zhang, H. Liu, J. Mi, L. Zhang, L. Liu, Y. Gong and B. Han

(c) The Author(s), under exclusive licence to Springer Nature Limited 2021

Oncogene (2021) 40:6140-6141; https://doi.org/10.1038/s41388-021-01995-z

Correction to: Oncogene https://doi.org/10.1038/onc.2017.380, published online 06 November 2017

Following the publication of this article an error was noted in Fig. 5k. The same transwell images were included for the Flag4B group and Flag $4 \mathrm{~B}+\mathrm{NC}$ group. The correct image has now been added for the Flag4B+NC group. The corrected version of Fig. 5 is included below. 
A

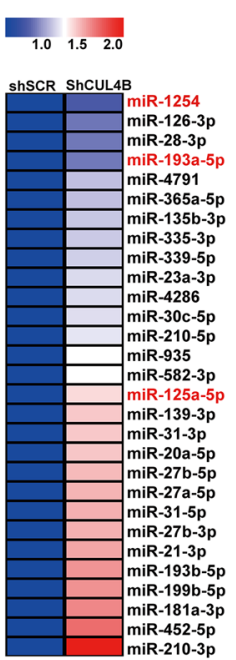

F

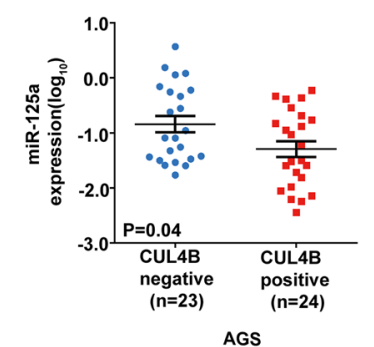

I

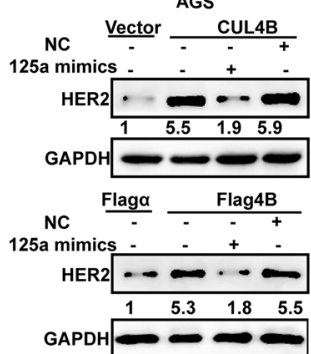

B

D
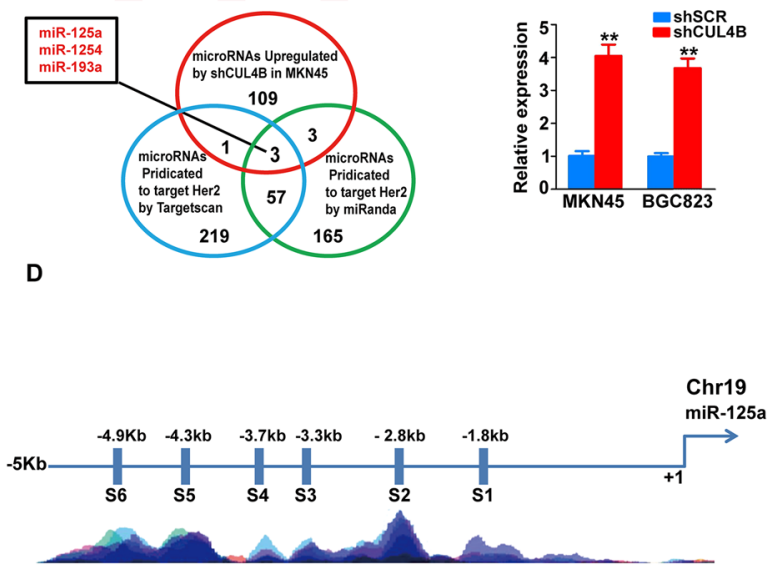

G
C

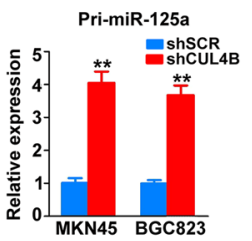

NC

125a mimics

Inhibitor NC
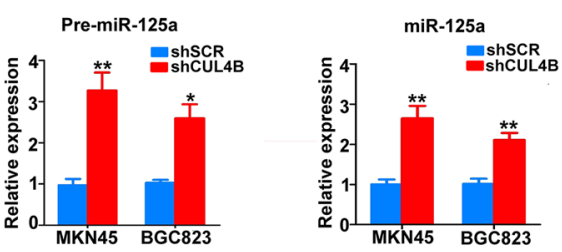

E $\quad 0.06$

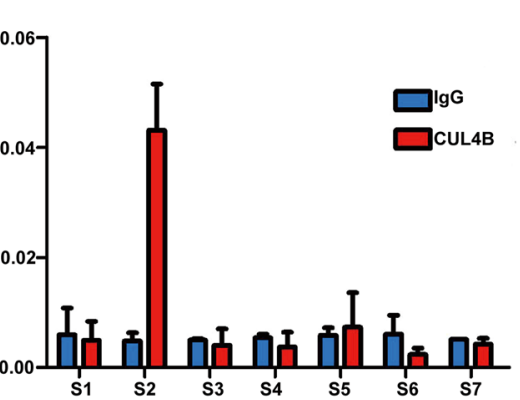

H
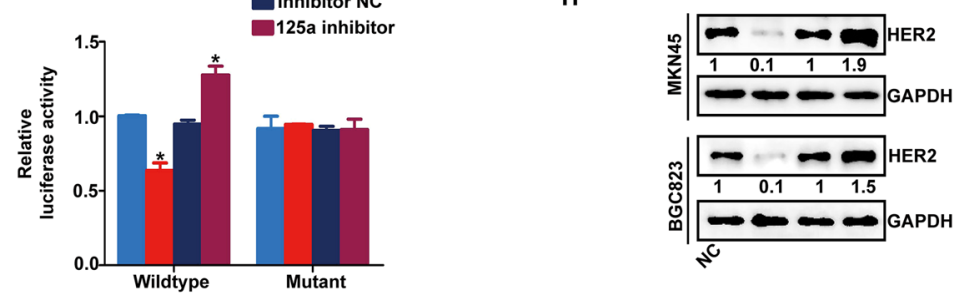

MKN45

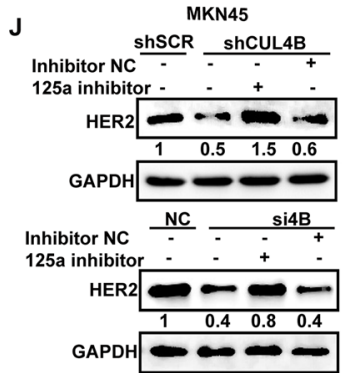

K
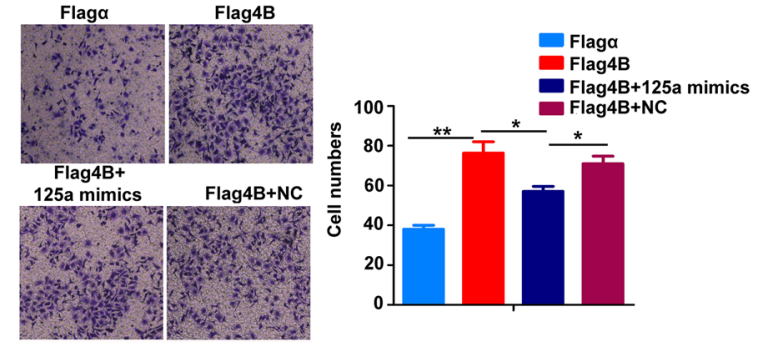

Fig. 5 CUL4B promotes HER2 expression via repressing miR-125a. a MiRNA profiling analysis of MKN45 cells in which CUL4B was knocked down compared with MKN45-shSCR cells. Pseudo-colors indicate expression levels from low to high (blue to red). shSCR: knockdown scramble control; shCUL4B: knockdown of CUL4B. b A Venn diagram depicting three miRNAs that were upregulated by CUL4B knockdown in MKN45 cells, predicted to target HER2 proteins by two prediction algorithms (TargetScan and miRanda). c Expression of primary and mature miR-125a expression was assessed by RT-qPCR in MKN45 and BGC823 cells. shRNA knockdown of CUL4B significantly increased expression levels of primary and mature miR-125a. ${ }^{*} P<0.05,{ }^{*} P<0.01$, based on Student's $t$-test. d Schematic diagram showing location of CUL4Bbinding sites of miR-125a regulatory region. S1, S2, S3, S4, S5 and S6 represented CUL4B-binding site, S7 was used as negative control and was located in the intron 4 of miR-125a. e ChIP assays with an anti-CUL4B or negative control (anti-lgG) antibodies showed CUL4B binding to the miR-125a promoter in MKN45 cells. The $y$ axis represents the \% input of the promoter fragments captured by the two different antibodies. S1-S6 regions and S7 were used as negative control. Site S2 of miR-125a carry binding sites for CUL4B, whereas no signal was detected at the miR-125a distal promoter (site S7). f MiR-125a expression was inversely correlated with CUL4B expression in human GC cases ( $n=47)$. These cases were categorized into CUL4B-positive and CUL4B-negative based on CUL4B expression level. The expression of miR-125a was measured by RT-qPCR assay and CUL4B-positive patients have lower miR-125a expression compared to CUL4B-negative patients. $\mathbf{g}$ HER2 is a direct target of miR-125a. Overexpression of miR-125a suppresses HER2 $3^{\prime}$-UTR but not mutant $3^{\prime}-$ UTR luciferase activities. ${ }^{*} P<0.05$, based on Student's $t$-test. $\mathbf{h}$ The protein levels of HER2 were assessed by western blot analysis in MKN45 and BGC823 cells transfected with miR-125a inhibitor, miR-125a mimics or negative control. Band intensity given underneath gel image was measured using ImageJ software, presented as fold change compared with controls for $\mathbf{h}-\mathbf{j}$, respectively. i AGS cells were stably/transiently transfected with indicated RNA and plasmids, respectively. Seventy-two hours later, CUL4B protein levels were determined by western blot. j MKN45 cells were stably/transiently transfected with indicated RNA and plasmids, respectively. Seventy-two hours later, CUL4B protein levels were determined by western blot. $\mathbf{k}$ AGS cells were transiently transfected with indicated RNA and plasmid. Forty-eight hours later migration ability of cells was examined by transwell migration assay. ${ }^{*} P<0.05,{ }^{* *} P<0.01$, based on Student's $t$-test. ChIP, chromatin immunoprecipitation. 